\title{
Efficient Simulation of Hybrid Stripmap/Spotlight SAR Raw Signals From Extended Scenes
}

\author{
Giorgio Franceschetti, Life Fellow, IEEE, Raffaella Guida, Student Member, IEEE, Antonio Iodice, Member, IEEE, \\ Daniele Riccio, Senior Member, IEEE, and Giuseppe Ruello, Member, IEEE
}

\begin{abstract}
The hybrid stripmap/spotlight mode for a synthetic aperture radar (SAR) system is able to generate microwave images with an azimuth resolution better than the one achieved in the stripmap mode and a ground coverage better than the one of the spotlight mode. In this paper, time- and frequencydomain-based procedures to simulate the raw signal in the hybrid stripmap/spotlight mode are presented and compared. We show that a two-dimensional Fourier domain approach, although highly desirable for its efficiency, is not viable. Accordingly, we propose a one-dimensional (1-D) range Fourier domain approach, followed by 1-D azimuth time-domain integration. This method is much more efficient than the time-domain one, so that extended scenes can be considered. In addition, it involves approximations usually acceptable in actual cases. Effectiveness of the simulation scheme is assessed by using numerical examples.
\end{abstract}

Index Terms-Scattering, simulation, synthetic aperture radar (SAR).

\section{INTRODUCTION}

$\mathbf{T}$ HE SYNTHETIC aperture radar (SAR) system can image an area over the ground in different operational modes: in the well-known stripmap mode, the radar antenna is pointed along a fixed direction with respect to the platform flight path and the antenna footprint covers a strip on the imaged surface as the platform moves (see Fig. 1). Accordingly, the extension of the illuminated area is theoretically unlimited in the along-track (azimuth) direction, but the azimuth resolution cannot be better than a half of the real antenna azimuth length.

With the spotlight configuration we can improve this resolution by increasing the synthetic aperture extension. The radar antenna beam is steered during the overall acquisition time (see Fig. 2), thus pointing always at the same area over the ground. Obviously, the achieved azimuth resolution improvement is traded off by the loss of ground coverage.

Most recently, a new operating mode, referred to as hybrid stripmap/spotlight mode, has been presented [1]-[3]. In the hybrid acquisition, the radar antenna beam is steered about a point farther away from the radar than the area being illuminated (see Fig. 3), thus allowing the generation of microwave images with

Manuscript received December 19, 2003; revised June 16, 2004.

G. Franceschetti is with the Dipartimento di Ingegneria Elettronica e delle Telecomunicazioni, Università di Napoli "Federico II," 80125 Naples, Italy and also with the University of California, Los Angeles, CA 90095 USA (e-mail: gfrance@unina.it).

R. Guida, A. Iodice, D. Riccio, and G. Ruello are with the Dipartimento di Ingegneria Elettronica e delle Telecomunicazioni, Università di Napoli “Federico II,"80125 Naples, Italy (e-mail: rafguida@unina.it; iodice@unina.it; daniele.riccio@unina.it; ruello@unina.it).

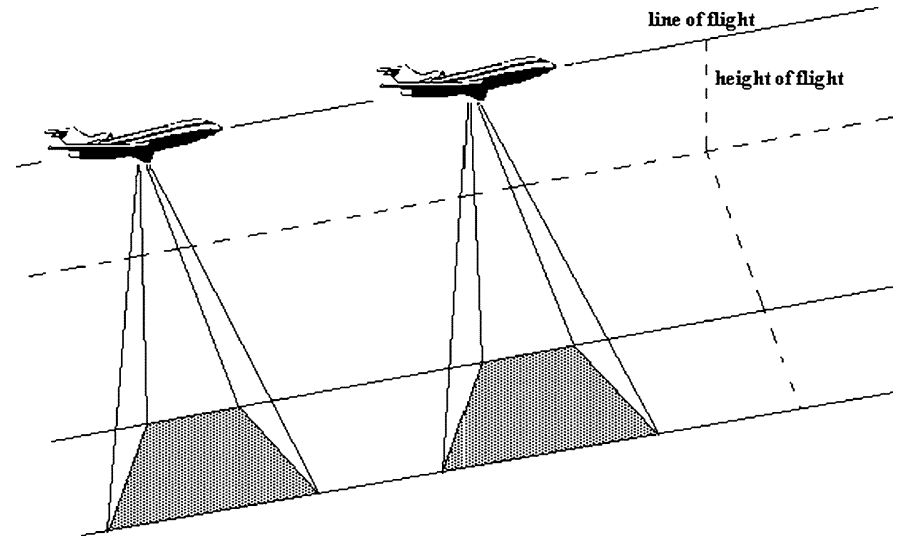

Fig. 1. Geometry of the stripmap mode.

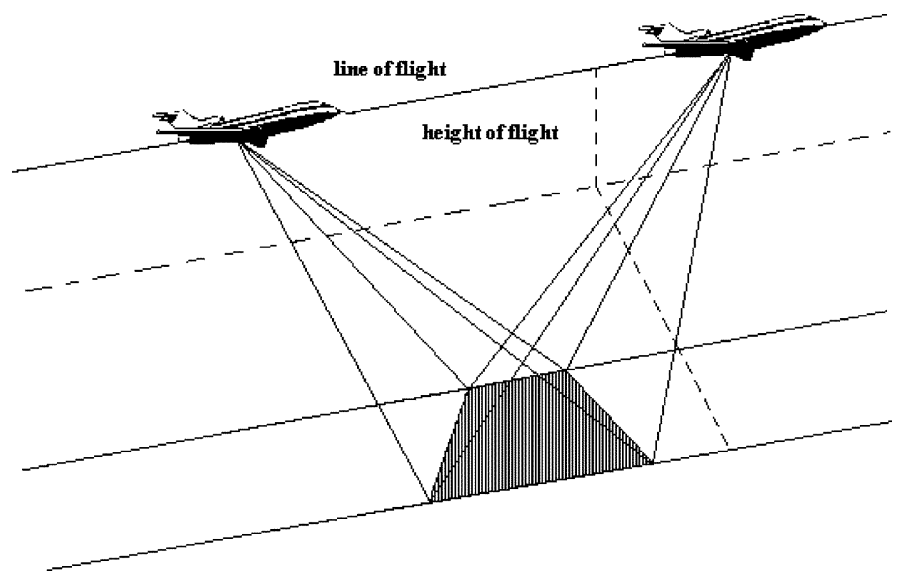

Fig. 2. Geometry of the spotlight mode.

an azimuth resolution better than that achieved in the stripmap configuration, and a ground coverage better than the one of the spotlight configuration.

A number of different processing procedures for hybrid mode have been proposed in the last years [1]-[4], and, although spaceborne SAR sensors operating in the hybrid mode are not yet available, some are currently under design, e.g., SAR 2000 within the Cosmo/Skymed project [5] or TerraSAR-X [6], [7]. In addition, airborne SAR sensors implementing hybrid mode are already available, e.g., the wideband phased-array SAR/MTI system PAMIR [8]. Therefore, the subject of design, processing, and data interpretation for the hybrid stripmap/spotlight SAR mode is gaining an increasingly wide interest in the remote sensing scientific community. 


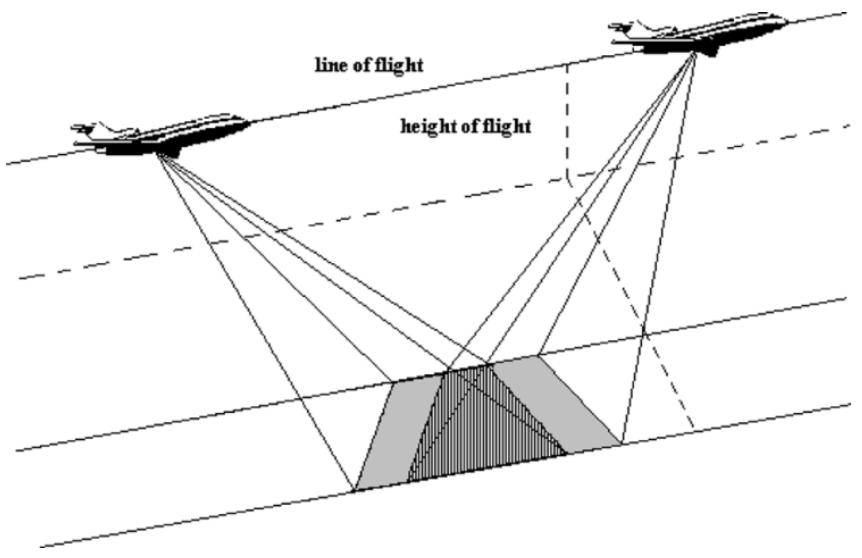

Fig. 3. Geometry of the hybrid stripmap/spotlight mode.

To quantitatively support the design of a SAR operating in the hybrid mode, to help mission planning, and to test processing algorithms, a SAR raw signal simulator is required, especially when real raw data are not available yet. In order to test the focusing capability of processing algorithms, point target simulators are usually sufficient. However, it is certainly useful to verify the effect of processing inaccuracies on simulated, and hence perfectly known, extended scenes: in fact, the effects of processing inaccuracies could be "masked" or "emphasized" when complex targets are considered, so that they can vary for different kinds of imaged scenes and hence for different applications. In addition, simulation of SAR raw signals from extended scenes is helpful in SAR system design and mission planning, because it allows to analyze the effects of different design and mission parameter choices for different kinds of imaged scenes. Accordingly, an extended scene SAR raw signal simulator is highly desirable. Such a simulator must include a scattering model and a radar model. The scattering model must evaluate the radar reflectivity as a function of the scene parameters (surface geometry and roughness, permittivity, and conductivity) and radar parameters (central frequency, bandwidth, altitude of flight, look-angle, polarization). The radar model must evaluate the raw signal that would be acquired onboard as a function of the radar reflectivity map as well as the sensor operating mode parameters (antenna dimensions, radiation diagrams and azimuthal electronic steering, spacecraft velocity, pulse bandwidth, pulse repetition frequency, sampling frequency).

Time-domain SAR raw signal simulation can be easily conceived, but it turns out to be enormously time and memory consuming when extended scenes are considered. The raw signal is represented by a twofold integral of reflectivity and impulse response function; such integral is not immediately recognized as a convolutional form, and its evaluation must be performed in the time domain. This is certainly not efficient, being the sampled reflectivity and impulse response function matrixes of comparable sizes.

Efficient simulators have been presented for the stripmap operational mode [9]-[11]. A frequency-domain approach can be followed: a space-variant transfer function is defined and evaluated in closed form; and an overall procedure is presented to efficiently evaluate the raw signal employing this transfer function, fast Fourier transforms (FFTs), and an appropriate grid deformation.
More recently, an efficient simulator has been presented for the spotlight operational mode [12]. Also, in this case, a frequency-domain approach can be followed to simulate the raw signal: an appropriately modified space-variant transfer function is defined and evaluated in closed form; and a different overall procedure is implemented that employs this modified transfer function, FFTs, appropriate grid deformation, and a simple final time-domain multiplication.

Conversely, to the best of our knowledge, no efficient extended scene SAR simulator for the hybrid mode is currently available. Only simple time-domain simulators, able to deal with point targets or small scenes, can be found in the literature [13], [14].

In this paper, efficient time- and frequency-domain-based procedures to simulate the raw signal in the hybrid stripmap/spotlight mode are presented and compared. To address this item, a new transfer function is defined. We show that in this case a two-dimensional (2-D) Fourier domain approach is not viable. However, we demonstrate that a one-dimensional (1-D) range Fourier domain approach, followed by 1-D azimuth time-domain integration, is possible, if we accept some approximations usually valid in the actual cases. We show that this method is still much more efficient than the time-domain one, so that extended scenes can be considered.

The paper is organized as follows. In Section II, the existing simulation schemes for the stripmap and spotlight configurations are briefly recalled, because they provide guidelines to deal with the hybrid case. In Section III, the hybrid configuration is considered, and the proposed simulation scheme is described. Its computational complexity is analyzed in Section IV, where simulation examples are also provided in order to assess its effectiveness. Finally, in Section V, some final remarks are reported.

\section{Simulation of SAR RaW Signals: STRIPMAP AND SPOTLIGHT MODES}

In this section, we evaluate the SAR raw signal for both the stripmap and the spotlight modes, both in time and frequency domains, in order to understand the efficient frequency-domain approaches proposed in [9]-[12] for the simulation of stripmap and spotlight SAR raw signals.

\section{A. Stripmap Mode}

Let us start from the stripmap mode. A chirp modulation of the transmitted pulse is assumed. The expression of the SAR raw signal is the following [15]:

$$
h_{\text {strip }}\left(x^{\prime}, r^{\prime}\right)=\iint \gamma(x, r) g_{\text {strip }}\left(x^{\prime}-x, r^{\prime}-r ; r\right) d x d r
$$

wherein

$$
\begin{aligned}
g_{\text {strip }}\left(x^{\prime}-x, r^{\prime}-r ; r\right)= & \exp \left[-j \frac{4 \pi}{\lambda} \Delta R\right] \\
& \cdot \exp \left[-j \frac{4 \pi}{\lambda} \frac{\frac{\Delta f}{f}}{c \tau}\left(r^{\prime}-r-\Delta R\right)^{2}\right] \\
& \cdot w^{2}\left(\frac{x^{\prime}-x}{X}\right) \operatorname{rect}\left[\frac{\left(r^{\prime}-r-\Delta R\right)}{\frac{c \tau}{2}}\right]
\end{aligned}
$$


is the SAR system impulse response, ${ }^{1}$ and

$$
\Delta R=\Delta R\left(x^{\prime}-x ; r\right)=R-r=\sqrt{r^{2}+\left(x^{\prime}-x\right)^{2}}-r .
$$

In (2.1)-(2.3) (see also Fig. 4), we have the following.

- $x, r$ and $\vartheta$ are the coordinates in the cylindrical coordinate system whose axis is the sensor line of flight.

- $A \equiv\left(x^{\prime}, 0,0\right)$ is the antenna position.

- $\gamma(x, r)$ is the scene reflectivity pattern ${ }^{2}$ including the phase factor $\exp [-j(4 \pi / \lambda) r]$.

- $\lambda$ and $f$ are, respectively, the carrier wavelength and frequency of the transmitted signal.

- $R$ is the distance from $A$ to the generic point $(x, r, \theta(x, r))$ of the scene.

- $\theta=\theta(x, r)$ is the soil surface equation.

- $R_{0}$ is the distance from the line of flight to the center of the scene.

- $\Delta f$ is the chirp bandwidth.

- $c$ is the speed of light.

- $\tau$ is the pulse duration time.

- $w(\cdot)$ is the azimuth illumination diagram of the real antenna over the ground.

- $X=\lambda R_{0} / L$ is the real antenna azimuth footprint (we assume that $w(\cdot)$ is negligible when the absolute value of its argument is larger than $1 / 2$, and that it is an even function).

- $L$ is the azimuth dimension of the real antenna.

- $\operatorname{rect}[t / T]$ is the standard rectangular window function, i.e., rect $[t / T]=1$ if $|t| \leq T / 2$; otherwise rect $[t / T]=0$.

- $r^{\prime}$ is $c / 2$ times the time elapsed from each pulse transmission.

If we ignore the $r$-dependence of $g_{\text {strip }}(\cdot)$, i.e., if we let $r=R_{0}$ in (2.3), then (2.1) is easily recognized as the 2-D convolution between $\gamma$ and $g_{\text {strip }}$ that can be efficiently performed in the 2-D Fourier-transformed (FT) domain. Even considering the $r$-dependence, (2.1) can be efficiently computed in the 2-D Fourier transformed domain: in fact, by using the stationary phase method, it can be shown [15] that the FT of (2.1) is ${ }^{3}$

$$
H_{\text {strip }}(\xi, \eta)=G_{0 \text { strip }}(\xi, \eta) \Gamma[\xi, \eta \Omega(\xi)+\mu(\xi)]
$$

where $H_{\text {strip }}(\xi, \eta)$ is the FT of $h_{\text {strip }}(x, r), \Gamma(\xi, \eta)$ is the FT of $\gamma(x, r)$

$$
\begin{aligned}
& G_{0 \text { strip }}(\xi, \eta)=\exp \left[j \frac{\eta^{2}}{4 b}\right] \exp {\left[j \frac{\xi^{2}}{4 a\left(1+\frac{\eta \lambda}{(4 \pi)}\right)}\right] } \\
& \cdot \operatorname{rect}\left[\frac{\eta}{\frac{2 b c \tau}{2}}\right] w^{2}\left(\frac{\xi}{2 a X}\right)
\end{aligned}
$$

\footnotetext{
${ }^{1}$ In (2.2), a factor rect $\left(x^{\prime} / X_{1}\right)$ should be added, accounting for the finite length of the considered raw data set. However, in the stripmap case, we always have $X_{1} \gg X$ and this rect factor can be neglected.

${ }^{2}$ Hereafter, we will assume $\gamma\left(x^{\prime}, x, r\right) \approx \gamma(x, r)$. Actually, the reflectivity pattern of still ground point changes as the sensor moves, but the approximation is acceptable for the distances involved.

${ }^{3}$ In this expression, as well as in (2.10) and (3.5), unessential multiplicative constants are ignored.
}

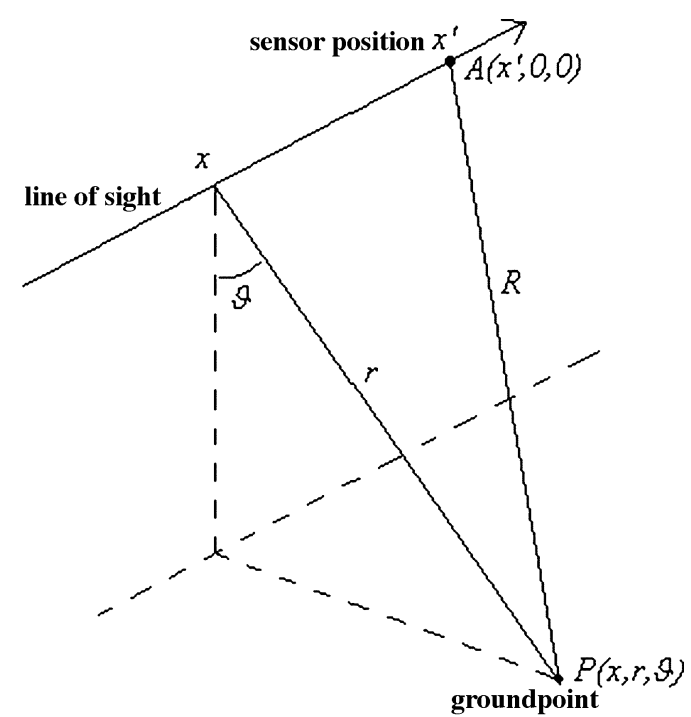

Fig. 4. Geometry of the problem.

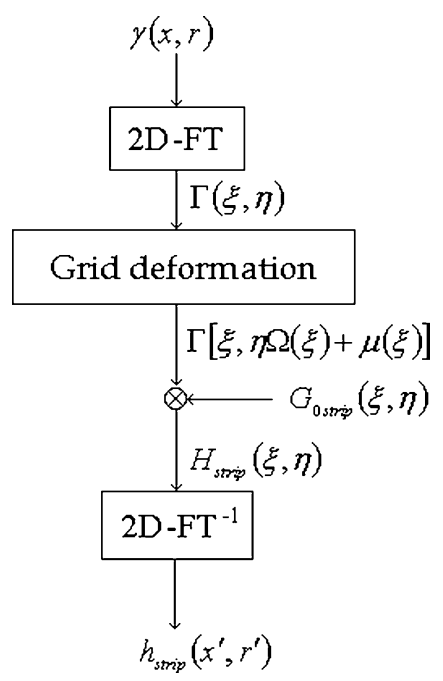

Fig. 5. Flowchart of stripmap SAR raw signal simulation.

is the FT of $g_{\text {strip }}\left(x^{\prime}-x, r^{\prime}-r ; r=R_{0}\right)$

$$
a=\frac{2 \pi}{\lambda R_{0}} \quad b=\frac{4 \pi}{\lambda} \frac{\frac{\Delta f}{f}}{c \tau}
$$

and the functions

$$
\mu(\xi)=\frac{\xi^{2}}{4 a R_{0}} \quad \Omega(\xi)=1-\frac{\xi^{2}}{4 a R_{0}} \frac{\lambda}{4 \pi}
$$

account for the $r$-space-variant characteristics of the SAR system, i.e., for the $r$-dependence of $g_{\text {strip }}(\cdot)$.

Equation (2.4) suggests that the stripmap SAR raw signal simulation can be performed as shown in the flowchart in Fig. 5, where the "Grid Deformation" block performs an interpolation in the Fourier domain, to obtain the desired values $\Gamma[\xi, \eta \Omega(\xi)+\mu(\xi)]$ from the available ones $\Gamma(\xi, \eta)$ (this step can be included in the 2-D FT block by using a chirp scaling algorithm [12], [16]). 
This is the method employed in the stripmap SAR raw signal simulator presented in [9]-[11]. Use of efficient FFT algorithms leads, in the case of extended scenes, to a processing time of different orders of magnitude smaller than the one required by a time-domain simulation directly based on (2.1)-(2.3).

\section{B. Spotlight Mode}

Let us now move to the spotlight mode. In this case, the SAR raw signal can be conveniently expressed as follows [12]:

$$
h_{\text {spot }}\left(x^{\prime}, r^{\prime}\right)=\operatorname{rect}\left[\frac{x^{\prime}}{X_{1}}\right] \widetilde{h}_{\text {spot }}\left(x^{\prime}, r^{\prime}\right)
$$

with

$$
\begin{aligned}
& \widetilde{h}_{\text {spot }}\left(x^{\prime}, r^{\prime}\right)=\iint \gamma(x, r) w^{2}\left(\frac{x}{X}\right) \\
& \cdot g_{\text {spot }}\left(x^{\prime}-x, r^{\prime}-r ; r\right) d x d r
\end{aligned}
$$

and

$$
\begin{aligned}
g_{\text {spot }}\left(x^{\prime}-x, r^{\prime}-r ; r\right)= & \exp \left[-j \frac{4 \pi}{\lambda} \Delta R\right] \\
& \cdot \exp \left[-j \frac{4 \pi}{\lambda} \frac{\frac{\Delta f}{f}}{c \tau}\left(r^{\prime}-r-\Delta R\right)^{2}\right] \\
& \cdot \operatorname{rect}\left[\frac{\left(r^{\prime}-r-\Delta R\right)}{\frac{c \tau}{2}}\right]
\end{aligned}
$$

where $X_{1}$ is the length of the trajectory flight portion used to acquire the raw data. Note that, due to the different acquisition geometry, in the spotlight case the antenna azimuth pattern $w(\cdot)$ depends on $x$ and not on the difference $x^{\prime}-x$ as in the stripmap case. In addition, (2.8) includes a rect $(\cdot)$ function of width $X_{1}$ accounting for the finite length of the trajectory flight portion used to acquire the raw data.

Again, apart from the $r$-dependence of $g_{\text {spot }}$, (that can be managed as in the stripmap case), the integral in (2.9) is easily recognized as the 2-D convolution between $\gamma(x, r) w^{2}(x / X)$ and $g_{\text {spot }}$, and can be efficiently evaluated in the 2-D Fourier transformed domain. In fact, it can be shown that the FT of (2.9) is [12], [15]

$$
\widetilde{H}_{\text {spot }}(\xi, \eta)=\widetilde{G}_{0 \text { spot }}(\xi, \eta) \bar{\Gamma}(\xi, \eta \Omega(\xi)+\mu(\xi))
$$

where $\bar{\Gamma}(\xi, \eta)$ is the FT of $\gamma(x, r) w^{2}(x / X)$ and

$$
\begin{aligned}
\widetilde{G}_{0 \text { spot }}(\xi, \eta)= & \exp \left[j \frac{\eta^{2}}{4 b}\right] \exp \left[j \frac{\xi^{2}}{4 a\left(1+\frac{\eta \lambda}{(4 \pi)}\right)}\right] \\
& \cdot \operatorname{rect}\left[\frac{\eta}{\frac{2 b c \tau}{2}}\right] \operatorname{rect}\left[\frac{\xi}{2 a\left(X+X_{1}\right)}\right]
\end{aligned}
$$

is the FT of $g_{\text {spot }}\left(x^{\prime}-x, r^{\prime}-r ; r=R_{0}\right)$.

Equations (2.8)-(2.12) suggest that the spotlight SAR raw signal simulation can be performed as shown in the flowchart in Fig. 6.

The above method is the one employed in the spotlight SAR raw signal simulator presented in [12]. In spite of the need for

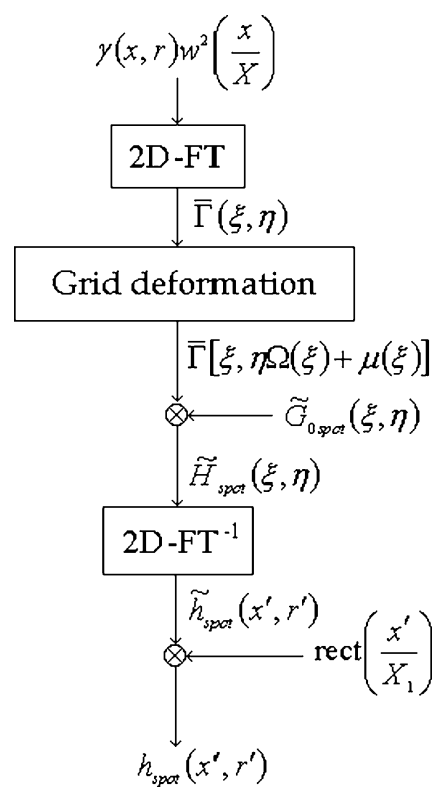

Fig. 6. Flowchart of spotlight SAR raw signal simulation.

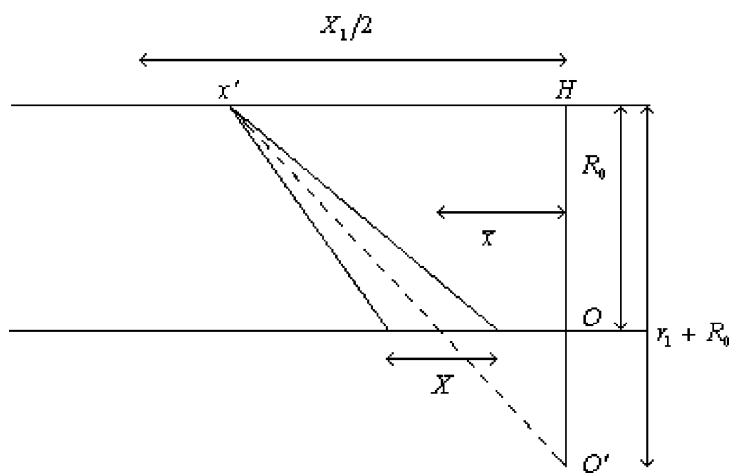

Fig. 7. Hybrid stripmap/spotlight mode. Illuminated area.

oversampling [12], also in this case use of efficient FFT algorithms leads, in the case of extended scenes, to a processing time of different orders of magnitude smaller than the one required by a time-domain simulation directly based on (2.8)-(2.10).

\section{SimUlation OF SAR RAW Signals: Hybrid STRIPMAP/SPOTLIGHT MODE}

In this section, we move to consider the hybrid stripmap/spotlight mode: we first evaluate its transfer function and then present a raw signal simulation procedure.

\section{A. Transfer Function}

In order to evaluate the SAR raw signal for the hybrid configuration, we have to introduce the factor [1]-[3]

$$
A=\frac{r_{1}}{r_{1}+R_{0}}
$$

where $R_{0}$ is the distance from the line of flight to the center of the scene, and $r_{1}$ is the distance from the ground to the beam steering point position beneath, so that $r_{1}+R_{0}$ is the distance from the line of flight to the steering point position (see Fig. 7). 
It can be shown [3] that use of the hybrid configuration is convenient when $2 X / X_{1}<A<1$, and that in this case the resolution is increased by a factor $1 / A$ with respect to the stripmap case, whereas the fully resolved covered area is increased by a factor $A\left(X_{1} / X\right)-1$ with respect to the spotlight case.

From the system geometry depicted in Fig. 7, it is evident that, by steering the antenna about infinity $(A=1)$ or about the scene center $(A=0)$, stripmap or spotlight configurations are obtained, respectively. In the intermediate cases $(0<A<1)$, for a given sensor position $x^{\prime}$, the illuminated area is centered around a point with azimuth coordinate $\bar{x}=A x^{\prime}$ and has an azimuth size equal to $X$ (see Fig. 7). Accordingly, the azimuth illumination diagram of the real antenna is of the form

$$
w\left(\frac{A x^{\prime}-x}{X}\right)
$$

that leads to the expression of the hybrid SAR raw signal

$h_{\text {hybrid }}\left(x^{\prime}, r^{\prime}\right)=\iint \gamma(x, r) g_{\text {hybrid }}\left(x^{\prime}-x, r^{\prime}-r ; x, r\right) d x d r$

where

$$
\begin{aligned}
g_{\text {hybrid }}\left(x^{\prime}-x, r^{\prime}-r ; x, r\right)= & \exp \left[-j \frac{4 \pi}{\lambda} \Delta R\right] \\
& \cdot \exp \left[-j \frac{4 \pi}{\lambda} \frac{\frac{\Delta f}{f}}{c \tau}\left(r^{\prime}-r-\Delta R\right)^{2}\right] \\
& \cdot w^{2}\left(\frac{A x^{\prime}-x}{X}\right) \operatorname{rect}\left[\frac{x^{\prime}}{X_{1}}\right] \\
& \cdot \operatorname{rect}\left[\frac{\left(r^{\prime}-r-\Delta R\right)}{\frac{c \tau}{2}}\right] .
\end{aligned}
$$

As expected, in the limiting cases $A=1$ and $A=0$, (3.3) and (3.4) reduce to the expression of the SAR raw signal in the stripmap and spotlight acquisition modes, respectively. Unfortunately, in the intermediate cases $(0<A<1)$, the integral in (3.3) cannot be expressed as a 2-D convolution and cannot be efficiently evaluated in the 2-D Fourier transformed domain. In fact, a stationary phase evaluation of the FT of (3.3) along the same guideline of the stripmap and spotlight cases [15] leads to

$$
\begin{aligned}
H_{\text {hybrid }}(\xi, \eta)=\iint \gamma(x, r) G_{\text {hybrid }}(\xi, \eta ; x, r) \\
\cdot \exp [-j \xi x] \exp [-j \eta r] d x d r
\end{aligned}
$$

where

$$
\begin{aligned}
G_{\text {hybrid }}(\xi, \eta ; x, r)= & \exp \left[j \frac{\eta^{2}}{4 b}\right] \exp \left[j \frac{\xi^{2}\left(\frac{r}{R_{0}}\right)}{4 a\left(1+\frac{\eta \lambda}{(4 \pi)}\right)}\right] \\
& \cdot \operatorname{rect}\left[\frac{\eta}{\frac{2 b c \tau}{2}}\right] \operatorname{rect}\left[\frac{\xi-2 a x}{2 a X_{1}}\right] \\
& \cdot w^{2}\left[\frac{\xi-2 a\left(1-\frac{1}{A}\right) x}{\frac{2 a X}{A}}\right]
\end{aligned}
$$

Examination of (3.5) and (3.6) shows that, at variance of previous cases, a twofold integration is needed. The $r$-integration can be dominated by expanding the second exponential in (3.6), as it is done in the strip and in the spot cases. The result would be a more involved functional dependence of the FT of $\gamma(x, r)$. But no similar procedure can be implemented for the $x$-dependence, and an efficient simulation algorithm cannot be devised in this domain. On the other hand, an algorithm in the space domain, directly based on (3.3), even if always possible in theory, is not computationally efficient, and hence not usable in practice if extended scenes are considered. Instead, we propose the approach that involves 1-D range FTs, described in Section III-B.

\section{B. Simulation Process}

Let us use the exact value of $\Delta R$ in the first exponential of (3.4), but let us approximate $\Delta R$ with its value at the scene center (i.e., at $r=R_{0}$ ) in the second exponential: in such a way, the most important part of the range space variance is accounted for, and only the effect of space variance on range curvature [15] is neglected. This is usually an acceptable approximation, as shown in the Appendix. If this is not the case, the range swath can be subdivided in different subswaths, for each of which above approximation holds (see the Appendix). By proceeding with this approximation, the hybrid SAR raw signal can be expressed as follows:

$$
\begin{aligned}
h_{\text {hybrid }}\left(x^{\prime}, r^{\prime}\right) \cong \int d x w^{2}\left[\frac{A x^{\prime}-x}{X}\right] \operatorname{rect}\left[\frac{x^{\prime}}{X_{1}}\right] \\
\cdot\left\{\int d r \gamma_{1}\left(x^{\prime}-x, x, r\right) g\left(x^{\prime}-x, r^{\prime}-r\right)\right\}
\end{aligned}
$$

where

$$
\begin{aligned}
\gamma_{1}\left(x^{\prime}-x, x, r\right)= & \gamma(x, r) \exp \left[-j \frac{4 \pi}{\lambda} \Delta R\right] \\
g\left(x^{\prime}-x, r^{\prime}-r\right)= & \exp \left[-j \frac{4 \pi}{\lambda} \frac{\frac{\Delta f}{f}}{c \tau}\left(r^{\prime}-r-\Delta R_{0}\right)^{2}\right] \\
& \cdot \operatorname{rect}\left[\frac{r^{\prime}-r-\Delta R_{0}}{\frac{c \tau}{2}}\right]
\end{aligned}
$$

and

$$
\Delta R_{0}=\Delta R\left(r=R_{0}\right)=\sqrt{R_{0}^{2}+\left(x^{\prime}-x\right)^{2}}-R_{0}
$$

thus separating the terms depending only on azimuth coordinate from those depending on both azimuth and range ones.

In (3.7), the last term in the graph parentheses is recognized as the range-convolution between $\gamma_{1}\left(x^{\prime}-x, x, r\right)$ and $g\left(x^{\prime}-x, r\right)$. Therefore, (3.7) can be also written as

$$
\begin{array}{r}
h_{\text {hybrid }}\left(x^{\prime}, r^{\prime}\right)=\int d x w^{2}\left[\frac{A x^{\prime}-x}{X}\right] \operatorname{rect}\left[\frac{x^{\prime}}{X_{1}}\right] \\
\cdot\left\{\Im^{-1}\left[\Gamma_{1}\left(x^{\prime}-x, x, \eta\right) \cdot G\left(x^{\prime}-x, \eta\right)\right]\right\}
\end{array}
$$

where $\Gamma_{1}\left(x^{\prime}-x, x, \eta\right)$ and $G\left(x^{\prime}-x, \eta\right)$ are the 1-D range FT of $\gamma_{1}\left(x^{\prime}-x, x, r\right)$ and $g\left(x^{\prime}-x, r\right)$, respectively

$$
\begin{aligned}
\Gamma_{1}\left(x^{\prime}-x, x, \eta\right)= & \int d r \gamma_{1}\left(x^{\prime}-x, x, r\right) \exp (-j \eta r) \\
G\left(x^{\prime}-x, \eta\right)= & \int d r \exp \left[-j \frac{4 \pi}{\lambda} \frac{\frac{\Delta f}{f}}{c \tau}\left(r-\Delta R_{0}\right)^{2}\right] \\
& \cdot \operatorname{rect}\left[\frac{\left(r-\Delta R_{0}\right)}{\frac{c \tau}{2}}\right] \exp (-j \eta r)
\end{aligned}
$$

and $\Im^{-1}$ stands for "inverse FT." 
Analytical evaluation of (3.13) is now in order. Letting

$$
q=r-\Delta R_{0}
$$

and

$$
\Psi(q)=\eta q+\frac{4 \pi}{\lambda} \frac{\frac{\Delta f}{f}}{c \tau} q^{2}
$$

then (3.13) becomes

$G\left(x^{\prime}-x, \eta\right)=\exp \left(-j \eta \Delta R_{0}\right) \int \operatorname{rect}\left[\frac{q}{\frac{c \tau}{2}}\right] \exp [-j \Psi(q)] d q$.

Normalization of $q$ to $c \tau / 2$ shows that stationary phase asymptotic evaluation of (3.16) is appropriate if $2 \pi \Delta f \cdot \tau$ is large, as it is the case for all SAR systems. The stationary phase point $q_{s}$ is solution of

$$
\left.\frac{\partial \psi}{\partial q}\right|_{q=q_{s}}=\eta+2 \frac{4 \pi\left(\frac{\Delta f}{f}\right)}{\lambda c \tau} q_{s}=0
$$

i.e.,

$$
q_{s}=-\frac{\eta}{2 b}
$$

and we get

$$
G\left(x^{\prime}-x, \eta\right) \approx \exp \left(-j \eta \Delta R_{0}\right) \exp \left[j \frac{\eta^{2}}{4 b}\right] \operatorname{rect}\left[\frac{\eta}{\frac{2 b c \tau}{2}}\right]
$$

where the parameter $b$ is given in (2.6).

Equations (3.11) and (3.19) suggest that the hybrid SAR raw signal simulation can be performed via the following steps (see Fig. 8).

Step 1) Generation of the scene reflectivity pattern $\gamma(x, r)$. This step is performed exactly in the same way as in the case of the stripmap mode: the scene surface is subdivided in facets smaller than the final system resolution, but much larger than wavelength; for each facet, the scattering coefficient is generated as a complex circular Gaussian random variable (characterized by Rayleigh amplitude and uniform phase distribution), whose variance is computed by considering incidence angle, polarization, and facet's roughness, conductivity, and permittivity (see [9] and [10] for details).

Step 2) For each $x^{\prime}$, perform the following:

- multiplication of $\gamma(x, r)$ by $\exp [-j(4 \pi / \lambda) \Delta R]$ to obtain $\gamma_{1}\left(x^{\prime}-x, x, r\right)$;

- implementation of 1-D FFT of $\gamma_{1}\left(x^{\prime}-x, x, r\right)$ to obtain $\Gamma_{1}\left(x^{\prime}-x, x, \eta\right)$;

- multiplication by $G\left(x^{\prime}-x, \eta\right)$;

- implementation of 1-D inverse FFT to get $\Im^{-1}\left[\Gamma_{1}\left(x^{\prime}-x, x, \eta\right) \cdot G\left(x^{\prime}-x, \eta\right)\right]$;

- multiplication by $w^{2}\left[\left(A x^{\prime}-x\right) / X\right]$;

- integration over $x$.

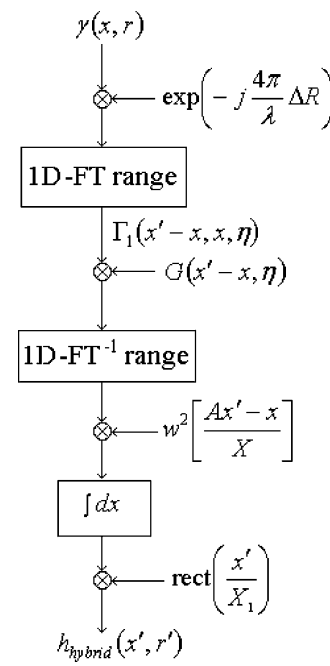

Fig. 8. Flowchart of hybrid stripmap/spotlight SAR raw signal simulation. All the steps must be iterated for each value of $x^{\prime}$.

Step 3) Multiplication by rect $\left[x^{\prime} / X_{1}\right]$.

This is the method employed in the simulator that we propose here.

It must be noted that presented procedure assumes a straight line flight path. This is usually a good approximation for a few kilometers portion of the elliptical orbit of a spaceborne sensor. Conversely, in the case of airborne sensors appreciable deviations from the ideal trajectory may occur: effects of these deviations can be easily accounted for by our simulation scheme, since the azimuth processing is performed in time domain. Accordingly, it is sufficient to let in [(3.4), (3.8), (3.10)]

$\Delta R=\Delta R\left(x^{\prime}-x ; r\right)=R-r=\sqrt{\left[r+\Delta r\left(x^{\prime}\right)\right]^{2}+\left(x^{\prime}-x\right)^{2}}-r$

where $\Delta r\left(x^{\prime}\right)$ is the projection along the line of sight of the deviation with respect to the nominal trajectory at the sensor azimuth location $x^{\prime}$.

\section{Performance Evaluation}

\section{A. Computational Complexity}

It is now appropriate to compare the computational complexity of the proposed algorithm to the one of a full time-domain direct approach. In this analysis, we do not consider generation of the reflectivity map, which is the same in both approaches.

If the hybrid raw signal is evaluated in time domain directly from (3.3), the efficiency of FFT codes is not exploited, and the computational complexity is

$$
N_{\mathrm{TD}} \approx\left(N_{x} N_{r}\right)^{2}
$$

wherein $N_{\mathrm{TD}}$ is the required number of complex multiplications and $N_{x}, N_{r}$ are the azimuth and range dimensions (in pixels) of the final hybrid raw signal, respectively (we are assuming that the number of scene facets is of the same order of the number of raw signal pixels). 
As for the Fourier approach, the 1-D range FFT of $\gamma_{1}\left(x^{\prime}-x, x, r\right)$ is calculated for each couple of values $x^{\prime}$ and $x$, so this step exhibits the computational complexity

$$
N_{x}^{2} \frac{N_{r}}{2} \log _{2} N_{r}
$$

At this point, the matrix $\Gamma_{1}\left(x^{\prime}-x, x, \eta\right)$ is multiplied by the function $G\left(x^{\prime}-x, \eta\right)$ for every value $\left(x^{\prime}, x, \eta\right)$, and then the inverse 1-D range FFT of the updated matrix is evaluated. This stage exhibits the computational complexity

$$
N_{x}^{2} N_{r}+N_{x}^{2} \frac{N_{r}}{2} \log _{2} N .
$$

Therefore, the overall computational complexity of the above described algorithm is

$$
N_{H}=N_{x}^{2} N_{r}\left(1+\log _{2} N_{r}\right)
$$

wherein $N_{H}$ is the number of complex multiplications. Accordingly, by using the suggested hybrid time-Fourier domain approach, processing time is reduced by the factor

$$
\frac{N_{H}}{N_{\mathrm{TD}}}=\frac{N_{x}^{2} N_{r}\left(1+\log _{2} N_{r}\right)}{N_{x}^{2} N_{r}^{2}}=\frac{1+\log _{2} N_{r}}{N_{r}}
$$

with respect to a time-domain simulation. For a $4096 \times 4096$ hybrid raw signal, we obtain a processing time decrease factor of about $1 / 315$.

\section{B. Simulation Examples}

Generation and examination of simulated data are necessary to test the effectiveness of the hybrid raw signal simulator. First of all, we want to verify that the raw signal corresponding to a single scattering point, simulated by using the proposed hybrid time-Fourier domain approach, is in agreement with the one obtained directly from the exact time-domain expression, i.e., (3.3) where the reflectivity map $\gamma(\cdot)$ is a Dirac pulse, so that no integration is needed.

We refer to a hypothetical hybrid spaceborne sensor, whose parameters are reported in Table I, third column. We simulate the raw signal of a point scatterer placed at the center of the illuminated scene (i.e., the coordinates of the point scatterer are $\left.x=0, r=R_{0}\right)$. First, phase error is considered, i.e., the phase difference between the raw signal simulated by using the proposed approach and the one obtained via full time-domain simulation: the results are shown in the plots of Fig. 9 for given values of $A$ and $Q=X_{1} / X$. In particular, in Fig. 9(a), the plot of a cut of this phase difference along the azimuth direction is reported, whereas in Fig. 9(b) the plot of a cut of the same phase difference along the range direction is shown. It can be noted that the absolute value of this phase difference is always smaller, and often much smaller, than $\pi / 10$, thus leading to negligible effects.

Raw signal amplitudes are considered in Fig. 10, where azimuth and range cuts of the amplitude of the raw signal obtained by the proposed approach and by using (3.3) are reported. Only small oscillations around the exact constant value can be noted.
TABLE I

MAIN SAR SYSTEM DATA USED IN THE SIMULATION RUNS

\begin{tabular}{l|r|r}
\hline Figure Number & 11 & $9-10-13-15$ \\
\hline Platform height $(h)$ & $5.7 \mathrm{~km}$ & $775 \mathrm{~km}$ \\
\hline Platform velocity $(v)$ & $0.08176 \mathrm{~km} / \mathrm{s}$ & $6.69 \mathrm{~km} / \mathrm{s}$ \\
\hline Look angle $(\theta)$ & 40 degrees & 23 degrees \\
\hline Azimuth antenna dimension $(L)$ & $1.0 \mathrm{~m}$ & $15.0 \mathrm{~m}$ \\
\hline Range antenna dimension & $0.12 \mathrm{~m}$ & $6.0 \mathrm{~m}$ \\
\hline Carrier frequency $(f)$ & $9.6 \mathrm{GHz}$ & $5.3 \mathrm{GHz}$ \\
\hline Pulse duration $(\tau)$ & $5 \mathrm{\mu s}$ & $55 \mu \mathrm{s}$ \\
\hline Pulse bandwidth $(\Delta f)$ & $100 \mathrm{MHz}$ & $70 \mathrm{MHz}$ \\
\hline Sampling frequency $\left(f_{\mathrm{c}}\right)$ & $100 \mathrm{MHz}$ & $100 \mathrm{MHz}$ \\
\hline Pulse repetition frequency $(\mathrm{PRF})$ & $300 \mathrm{~Hz}$ & $900 \mathrm{~Hz}$ \\
\hline A & 0.80 & 0.50 \\
\hline $\mathrm{Q}$ & 3 & 6 \\
\hline
\end{tabular}

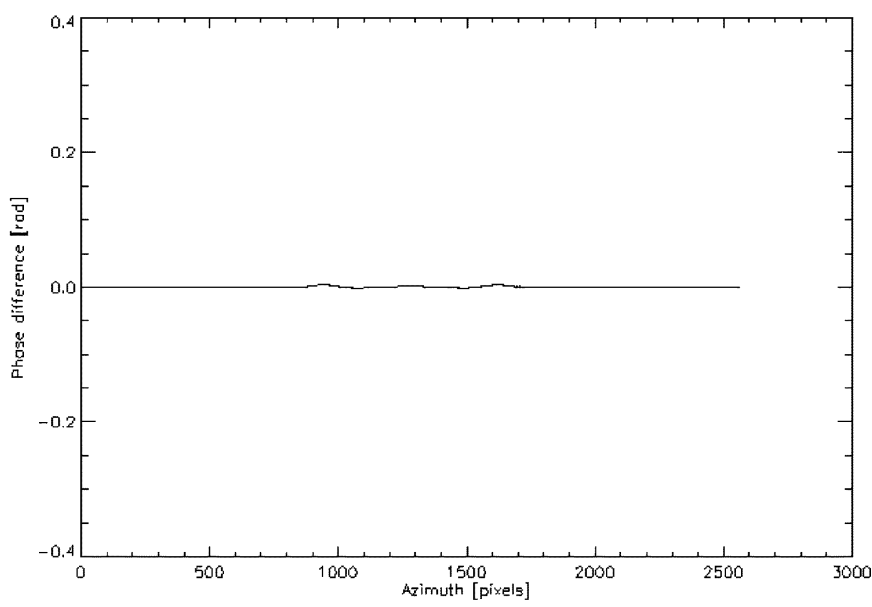

(a)

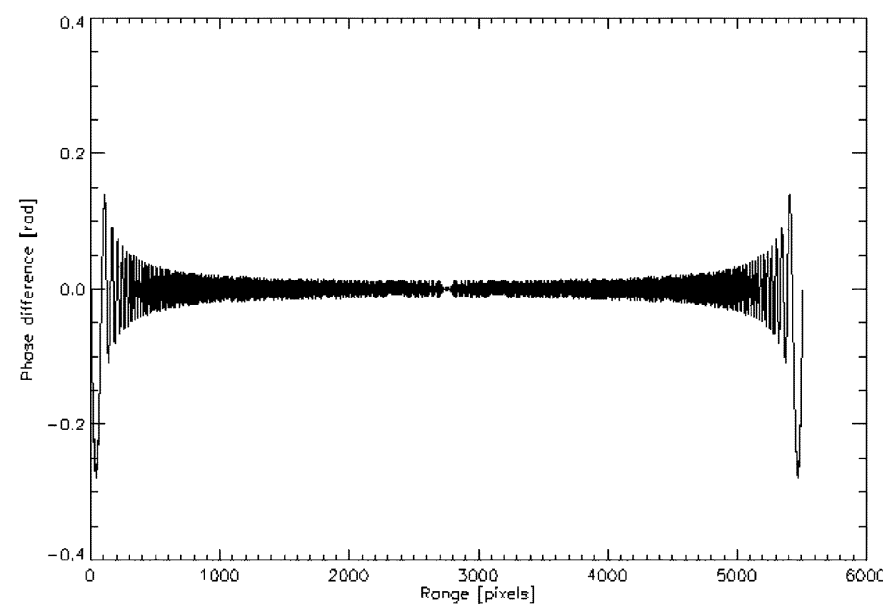

(b)

Fig. 9. Difference between the phase of the raw signal simulated by using the proposed approach and the phase of the raw signal obtained by time-domain simulation. (a) Azimuth cut. (b) Range cut. $A=0.50, Q=6$. The scattering point is placed in the center of the illuminated scene.

Similar comparisons for a point scatterer located at the azimuth and at the range borders of the illuminated area and for different values of the factors $A$ and $Q$ provide very similar results: the error is essentially the same, thus validating the proposed approach.

An airborne case (see Table I, second column) with instable trajectory is now analyzed. By using the approach described at the end of Section III-B we can deal with extended scenes 


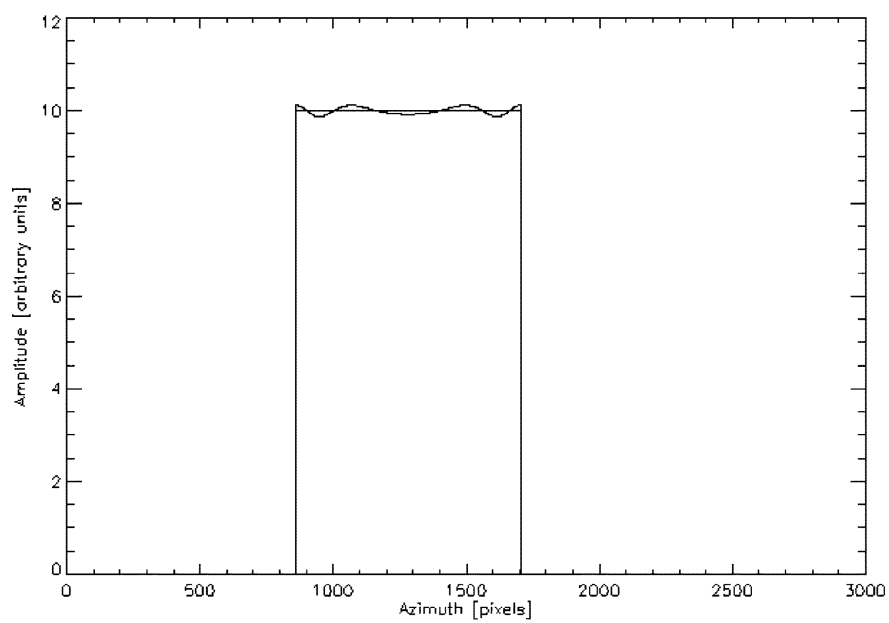

(a)

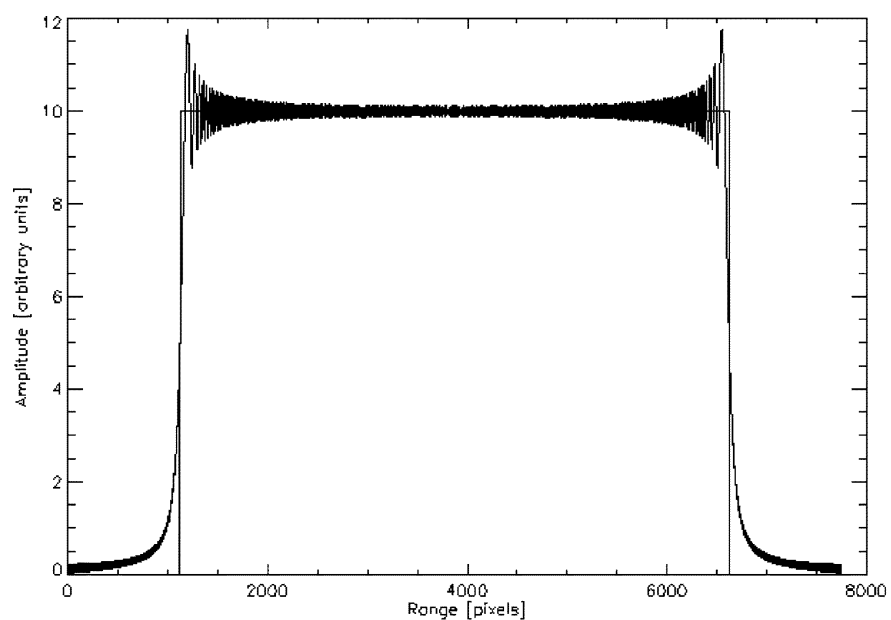

(b)

Fig. 10. Amplitudes of the raw signals simulated by using (oscillating curve) the proposed approach and (straight line) obtained by time-domain simulation. (a) Azimuth cut. (b) Range cut. $A=0.50, Q=6$. The scattering point is placed in the center of the illuminated scene.

and arbitrary trajectory deviations. However, we here consider a scattering point located at the center of the illuminated scene and sinusoidal deviations with respect to the ideal trajectory. In fact, in this case the effect of trajectory instability on the final image can be theoretically predicted [17], and it consists of the appearance of replicas weighted by factors related to Bessel functions and with a spatial separation related to the spatial period $P$ of the sinusoidal deviation. More precisely, the spatial separation is expected to be [17]

$$
D x=\left(\frac{L}{2}\right)\left(\frac{X}{P}\right) .
$$

In our simulation, the trajectory sinusoidal deviation has a 1-cm amplitude and a 11.6-m period. In Fig. 11, an azimuth cut of the image obtained by processing the simulated raw signal with no motion compensation is shown. As expected, a number of weighted replicas of the point target appear. The minimum visible spacing in Fig. 11 is about 36 pixels, which, multiplied by the azimuth pixel spacing $(0.28 \mathrm{~m})$, gives a $10-\mathrm{m}$ spacing. This value is in agreement with the expected one, obtained by

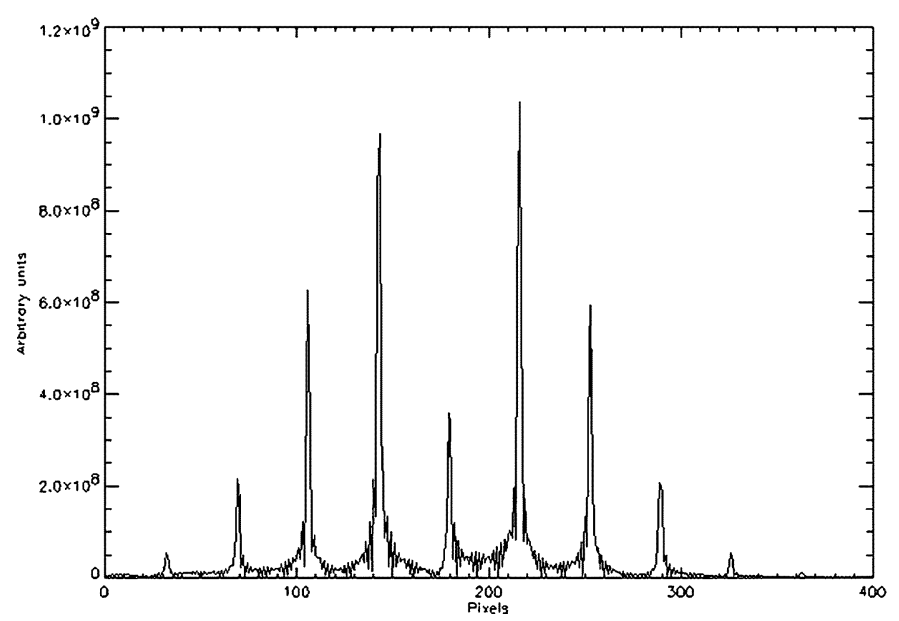

Fig. 11. Azimuth cut of the image obtained by processing the simulated raw signal relative to an airborne SAR system with sinusoidal trajectory deviation (amplitude $=1 \mathrm{~cm}$, period $=11.6 \mathrm{~m}) . A=0.80, Q=3$. The scattering point is placed in the center of the illuminated scene.

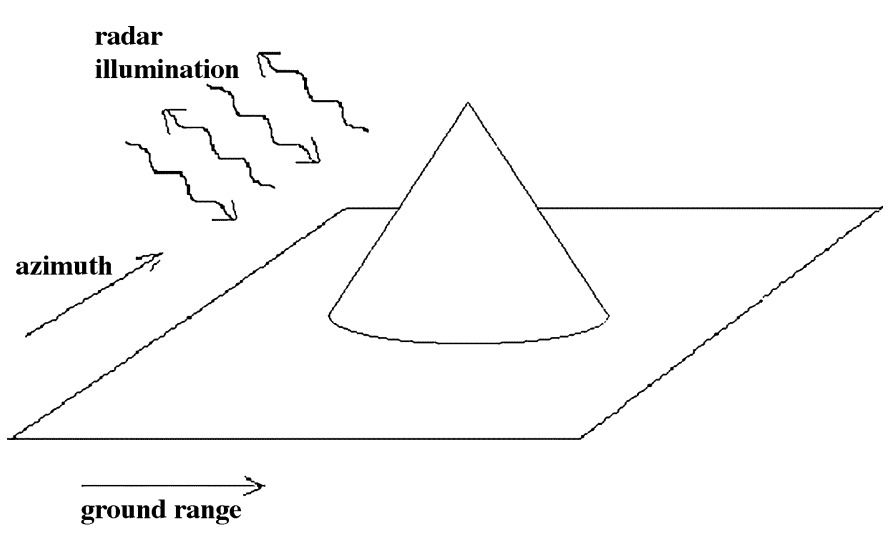

Fig. 12. "Canonical" extended scene: a cone over a plane.

using in (4.6) the airborne system data of Table $\mathrm{I}(X=232.5 \mathrm{~m}$, $L=1 \mathrm{~m})$ and $P=11.6 \mathrm{~m}$.

Simulations relevant to extended scenes are now in order. We consider the same spaceborne SAR system data of Table I, third column, and a "canonical" extended scene: a cone over a flat plane (see Fig. 12). In the following experiments, we assume that outside the fully resolved area the scene is perfectly absorbing. Corresponding raw signal has been generated. In Fig. 13(a), we show the image that can be obtained by using a hybrid focusing algorithm, whereas in Fig. 13(b) we show the image obtained by processing the simulated raw signals with a Fourier domain focusing algorithm conceived for stripmap raw signals. In both cases, near range is on the left. Data relevant to Fig. 13 are reported in Table II, third column. Note that in Fig. 13(b) the azimuth spectrum folding effect, due to the fact that the azimuth raw signal bandwidth is greater than the PRF, causes the appearance of some replicas of the imaged scene, since a Fourier Domain stripmap focusing algorithm has been used. This is in agreement with what happens for real hybrid data. The upper and lower replicas, related to the azimuth borders of the raw signal, exhibit dark areas due to the fact that outside the fully resolved area the scene is perfectly absorbing. 


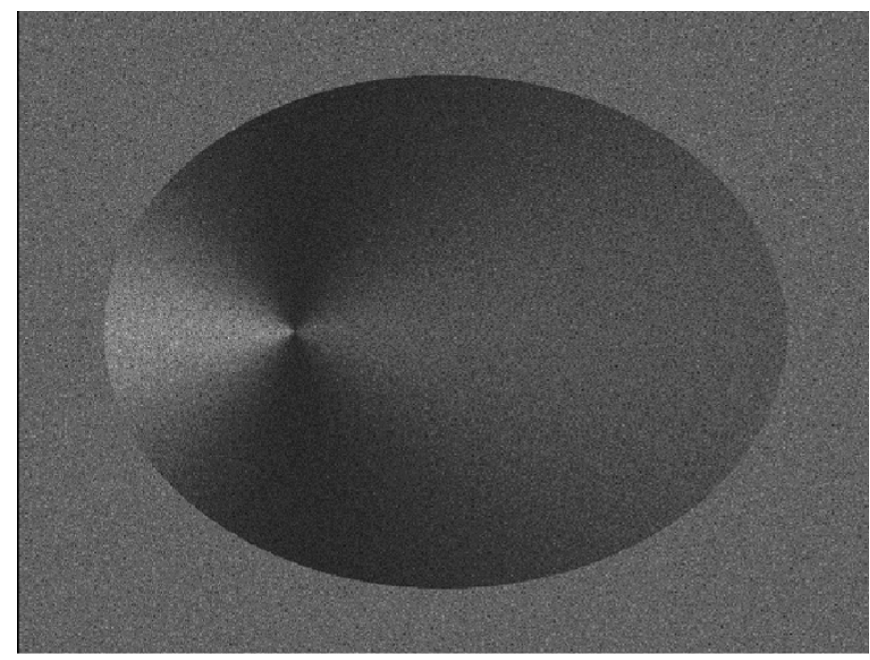

(a)

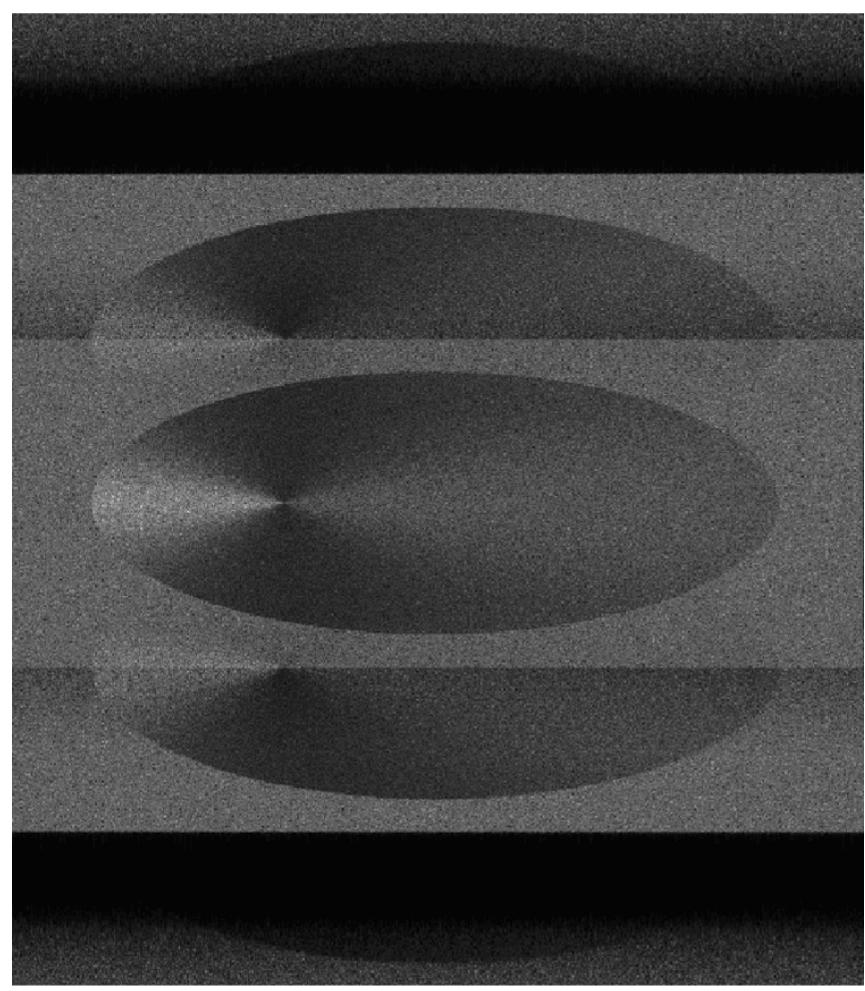

(a)

Fig. 13. Image of a cone over a plane obtained by (a) ideal hybrid processing and (b) by processing the simulated hybrid SAR raw signal via a Fourier domain stripmap focusing algorithm. Near range is on the left. $A=0.50, Q=6$. Azimuth spectrum folding effect is evident in (b). In (a), the azimuth extension of the scene appears greater than the one of (b) because of the better resolution of the hybrid mode that leads to a larger number of pixels.

Finally, we consider an actual complex extended scene, given by the digital elevation model of the area of Maratea, Italy (see Fig. 14). Corresponding raw signal has been generated with the same system parameters of Figs. 12 and 13. The final images are shown in Fig. 15 according to the same format of Fig. 13. Considerations analogous to those reported for Fig. 13 can be repeated for Fig. 15, but in Fig. 15(b) the effects of inappropriate processing are dramatically emphasized in this more complex scene.
TABLE II

MAIN Scene Data RELEVANT To Figs. 9-15

\begin{tabular}{l|r|r}
\hline Figure Number & 11 & $9-10-13-15$ \\
\hline Azimuth dimension of the scene $(\mathrm{km})$ & 0.3 & 6.4 \\
\hline Ground range dimension of the scene $(\mathrm{km})$ & 1.3 & 8.6 \\
\hline Azimuth resolution $(\mathrm{m})$ & 0.4 & 3.8 \\
\hline Ground range resolution (m) & 2.3 & 3.8 \\
\hline Raw signal azimuth size (pixels) & 2560 & 2565 \\
\hline Raw signal range size (pixels) & 1048 & 7750 \\
\hline
\end{tabular}

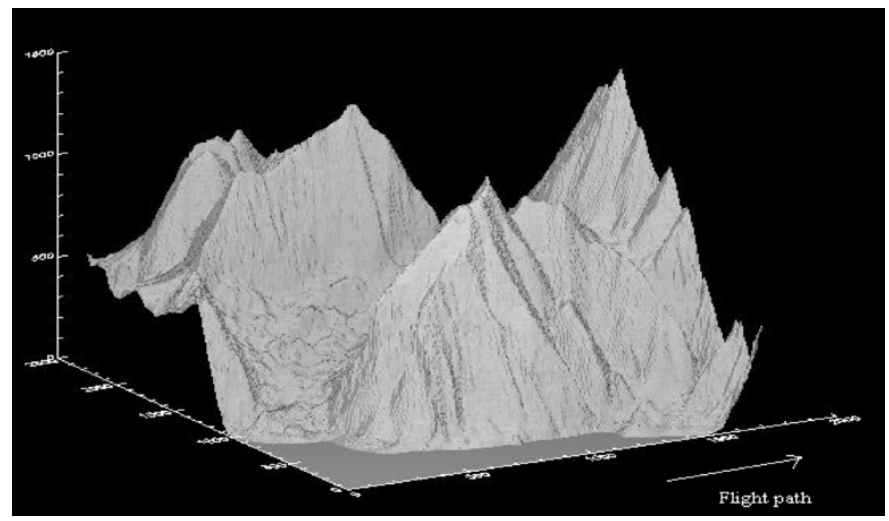

Fig. 14. "Actual" extended scene. The digital elevation model of the area of Maratea, Italy.

A few last words are now due about processing times. Obviously, each raw signal simulation required a processing time depending on the chosen system parameters (Table I) and couple $(A, Q)$. In any case, for scenes of the order of some thousands by some thousands pixels, processing time is of the order of several hours. For instance, for a scene of $3650 \times 3650$ samples, the raw signal simulation took about $11 \mathrm{~h}$ on a Pentium IV 2-GHz personal computer. Note that time-domain processing for the same scene would require a processing time of the order of months.

\section{CONCLUSION}

In this paper, time-domain and hybrid time-frequency-domain-based procedures to simulate the raw signal in the hybrid stripmap/spotlight mode have been presented and compared. A new simulation scheme has been proposed, based on a 1-D range Fourier domain approach followed by 1-D azimuth time-domain integration. This method has been shown to be much more efficient than the full time-domain one, so that extended scenes can be considered. In addition, it has been verified that the method involves approximations usually acceptable in the actual cases.

Effectiveness of the simulator has been verified by comparing simulated raw signal corresponding to a single scattering point (placed at different positions in the illuminated scene) to the corresponding available time-domain exact expression.

Hybrid SAR raw signals corresponding to extended canonical and actual scenes have been also simulated. Results confirm the consistency of the proposed simulation scheme and allow to highlight some interesting properties of the hybrid stripmap/spotlight SAR signals. 


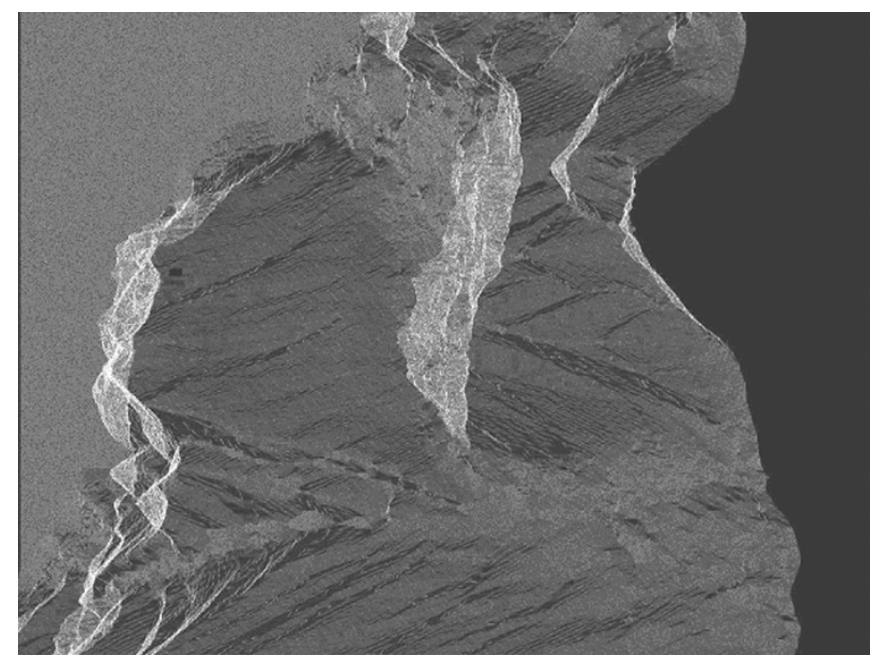

(a)

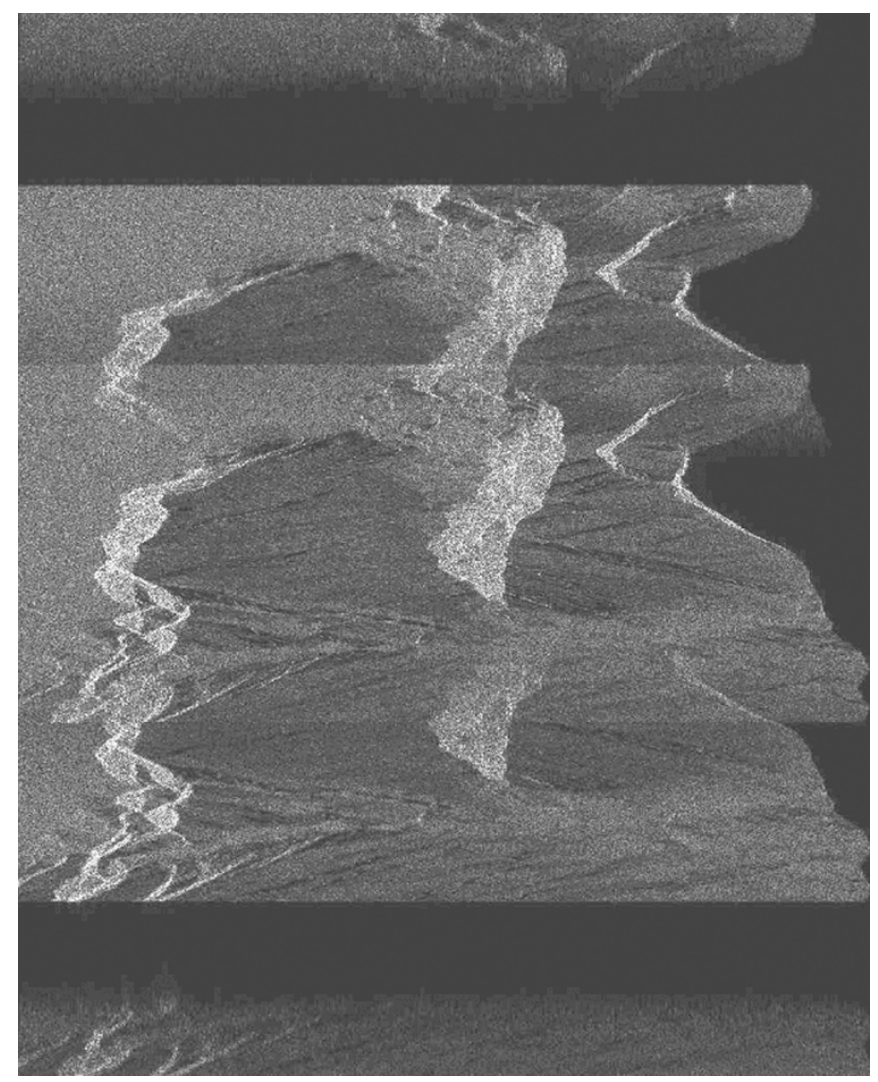

(b)

Fig. 15. Image of an actual complex scene (Maratea), obtained by (a) ideal hybrid processing and (b) by processing the simulated hybrid SAR raw signal via a Fourier domain stripmap focusing algorithm. Near range is on the left. $A=0.50, Q=6$. Azimuth spectrum folding effect is evident in (b).

\section{APPENDIX}

In this Appendix, we determine the conditions under which the approximation involved in (3.7) holds, i.e.,

$\exp \left[-j \frac{4 \pi}{\lambda} \frac{\frac{\Delta f}{f}}{c \tau}\left(r^{\prime}-r-\Delta R\right)^{2}\right]$

$$
\cong \exp \left[-j \frac{4 \pi}{\lambda} \frac{\frac{\Delta f}{f}}{c \tau}\left(r^{\prime}-r-\Delta R_{0}\right)^{2}\right]
$$

where $\Delta R_{0}$ is given by (3.10).

First of all, we note that

$$
\begin{aligned}
\left(r^{\prime}-r-\Delta R\right)^{2}= & \left(r^{\prime}-r-\Delta R_{0}\right)^{2}-2\left(r^{\prime}-r-\Delta R_{0}\right) \\
& \cdot\left(\Delta R-\Delta R_{0}\right)+\left(\Delta R-\Delta R_{0}\right)^{2} \\
= & \left(r^{\prime}-r-\Delta R_{0}\right)^{2}-2\left(r^{\prime}-r-\Delta \bar{R}\right) \\
& \cdot\left(\Delta R-\Delta R_{0}\right)
\end{aligned}
$$

with $\Delta \bar{R}=\left(\Delta R+\Delta R_{0}\right) / 2$. Accordingly, (A1) holds if

$$
\frac{4 \pi}{\lambda} \frac{\frac{\Delta f}{f}}{c \tau} 2\left(r^{\prime}-r-\Delta \bar{R}\right)\left(\Delta R-\Delta R_{0}\right) \ll 1
$$

In practice, it is sufficient that above quantity is smaller than $\pi / 4$ in the worst case (i.e., in near or far range).

Computation of upper bounds for the two factors appearing in (A3) is in order.

For the first, we have $\left(r^{\prime}-r-\Delta \bar{R}\right) \cong\left(r^{\prime}-r-\Delta R\right) \leq c \tau / 2$. For the second, we have

$$
\begin{aligned}
\Delta R-\Delta R_{0} & \cong \frac{\left(x^{\prime}-x\right)^{2}}{2 r}-\frac{\left(x^{\prime}-x\right)^{2}}{2 R_{0}} \\
& =\frac{\left(x^{\prime}-x\right)^{2}}{2 R_{0}}\left(\frac{R_{0}}{r}-1\right) \cong-\frac{\left(x^{\prime}-x\right)^{2}}{2 R_{0}^{2}}\left(r-R_{0}\right)
\end{aligned}
$$

and

$$
\begin{aligned}
& \left|x^{\prime}-x\right| \leq \frac{X_{1}-X_{F}}{2} \\
& \left|r-R_{0}\right| \leq \frac{S}{2}
\end{aligned}
$$

where $X_{F}=A X_{1}-X$ is azimuth size of the fully resolved zone [3], and $S$ is the (slant) range extension of the imaged area. It follows that

$$
\Delta R-\Delta R_{0} \leq \frac{\left(X_{1}-X_{F}\right)^{2}}{2 R_{0}^{2} \cdot 4} \cdot \frac{S}{2}=\frac{1}{8}\left(\frac{X_{1}-X_{F}}{R_{0}}\right)^{2} \cdot \frac{S}{2}
$$

The final result is

$\frac{4 \pi}{\lambda} \frac{\frac{\Delta f}{f}}{c \tau} 2\left(r^{\prime}-r-\Delta \bar{R}\right)\left(\Delta R-\Delta R_{0}\right) \leq \frac{\pi \Delta f}{c} \frac{1}{2}\left(\frac{X_{1}-X_{F}}{R_{0}}\right)^{2} \cdot \frac{S}{2}$.

The factor $\pi \Delta f /(2 c)$ is usually of the order of $10^{-1}$, approaching 1 only for very high range resolution systems (of the order of $1 \mathrm{~m}$ ). The ratio in parenthesis is of the order of a few times $10^{-2}$, so that its square is of the order of $10^{-3}$ or $10^{-4}$. Accordingly, even in the case of very high range resolution, a range swath of the order of a few kilometers is 
allowed. For instance, for a hypothetic high-resolution spaceborne SAR system with $\lambda=3 \mathrm{~cm}, L=6 \mathrm{~m}, R_{0}=1000 \mathrm{~km}$ (so that $X=5 \mathrm{~km}$ ), $\Delta f=150 \mathrm{MHz}, X_{1}=30 \mathrm{~km}$, and $A=0.5$ we have

$$
\frac{\pi \Delta f}{c} \frac{1}{2}\left(\frac{X_{1}-X_{F}}{R_{0}}\right)^{2}=\frac{\pi}{4} \cdot \frac{1}{2500} \mathrm{~m}^{1}
$$

so that a range swath of about $5 \mathrm{~km}$ is allowed. Considering that in this case the azimuth size of the fully resolved scene is $X_{F}=10 \mathrm{~km}$, the obtained range swath is realistic. In any case, a wider range swath of $10 \mathrm{~km}$ can be simulated by generating separately the raw signals from two adjacent range swaths and properly summing them.

\section{REFERENCES}

[1] D. P. Belcher and C. J. Baker, "High resolution processing of hybrid stripmap/spotlight mode SAR," Proc. Inst. Elect. Eng., Radar Sonar Navigat., vol. 143, pp. 366-374, 1996.

[2] G. Fornaro, R. Lanari, E. Sansosti, F. Serafino, and S. Zoffoli, "A new algorithm for processing hybrid strip-map/spotlight mode synthetic aperture radar data," Proc. SPIE, vol. 4173, pp. 17-28, 2000.

[3] R. Lanari, S. Zoffoli, E. Sansosti, G. Fornaro, and F. Serafino, "New approach for hybrid strip-map/spotlight SAR data focusing," Proc. Inst. Elect. Eng., Radar Sonar Navigat., vol. 148, pp. 363-372, 2001.

[4] J. Mittermayer, R. T. Lord, and E. Boerner, "Sliding spotlight SAR processing for TerraSAR-X using a new formulation of the extended chirp scaling algorithm," in Proc. IGARSS, Toulose, France, 2003, pp. 1462-1464.

[5] F. Caltagirone, P. Spera, R. Vigliotti, and G. Manoni, "'SkyMed/COSMO mission overview," in Proc. IGARSS, Seattle, WA, 1998, pp. 683-685.

[6] M. Suess, S. Riegger, W. Pitz, and R. Werninghaus, "TERRASAR-XDesign and performance," in Proc. EUSAR, Koln, Germany, 2002.

[7] J. Mittermayer, V. Alberga, S. Buckreuß, and S. Riegger, "TerraSAR-X: Predicted performance," Proc. SPIE, vol. 4881, 2002.

[8] J. H. G. Ender and A. R. Brenner, "PAMIR-A wideband phased array SAR/MIT system," Proc. Inst. Elect. Eng., Radar Sonar Navigat., vol. 150, pp. 165-172, 2003.

[9] G. Franceschetti, M. Migliaccio, D. Riccio, and G. Schirinzi, "SARAS: A SAR raw signal simulator," IEEE Trans. Geosci. Remote Sensing, vol. 30, pp. 110-123, Jan. 1992.

[10] G. Franceschetti, M. Migliaccio, and D. Riccio, "SAR simulation of actual ground sites described in terms of sparse input data," IEEE Trans. Geosci. Remote Sensing, vol. 32, pp. 1160-1169, Nov. 1994.

[11] G. Franceschetti, A. Iodice, M. Migliaccio, and D. Riccio, "A novel across-track SAR interferometry simulator," IEEE Trans. Geosci. Remote Sensing, vol. 36, pp. 950-962, May 1998.

[12] S. Cimmino, G. Franceschetti, A. Iodice, D. Riccio, and G. Ruello, "Efficient spotlight SAR raw signal simulation of extended scenes," IEEE Trans. Geosci. Remote Sensing, vol. 41, pp. 2329-2337, Oct. 2003

[13] E. Boerner, R. T. Lord, J. Mittermayer, and R. Bamler, "Evaluation of TerraSAR-X spotlight processing accuracy based on a new spotlight raw data simulator," in Proc. IGARSS, Toulose, France, 2003, pp. $1323-1325$

[14] A. Mori and F. D. Vita, "A raw signal simulator for stripmap, spotlight and hybrid mode interferometric SAR on spaceborne platform," in Proc. SRL Science Mission Meeting: What's Next?, Florence, Italy, 2001.

[15] G. Franceschetti and R. Lanari, Synthetic Aperture Radar Processing. Boca Raton, FL: CRC, 1999.

[16] R. K. Raney, H. Runge, R. Bamler, I. G. Cumming, and F. H. Wong, "Precision SAR processing using chirp scaling," IEEE Trans. Geosci. Remote Sensing, vol. 32, pp. 786-799, July 1994.
[17] G. Fornaro, "Trajectory deviations in airborne SAR: Analysis and compensation," IEEE Trans. Aerosp. Electron. Syst., vol. 35, pp. 997-1009, July 1999.

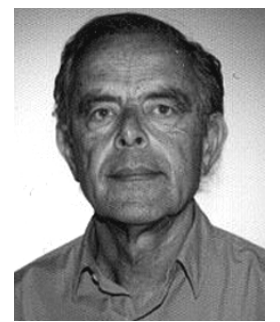

Giorgio Franceschetti (S'60-M'62-SM'85-F'88LF'01) was born in Italy.

He is currently a Full Professor of electromagnetic wave theory at University of Naples, Naples, Italy, since 1968. He is also an Adjunct Professor at the University of California, Los Angeles (UCLA) and a Distinguished Visiting Scientist at the Jet Propulsion Laboratory, California Institute of Technology (Caltech), Pasadena. He was a Visiting Professor at the University of Illinois, Urbana-Champaign, in 1976 and 1977, and at UCLA in 1980 and 1982 He was a Research Associate at Caltech, in 1981 and 1983, a Visiting Professor at National Somalia University, Mogadishu, Somalia, in 1984, and a Visiting Professor at the University of Santiago de Compostela, Santiago de Compostela, Spain, in 1995. He has published several books and more than 140 refereed papers in the field of applied electromagnetics (reflector antennas, transient phenomena, shielding, nonlinear propagation, and scattering) and, more recently, in the field of SAR data processing and simulation. He has lectured in several summer schools in China, the United Kingdom, Holland, Italy, Spain, Sweden, and the United States.

Dr. Franceschetti was a Fulbright Scholar at Caltech in 1973. He has been the recipient of several national and international awards. He was Director of IRECE, a Research Institute of the Italian National Council of Research (CNR), and member of the board of the Italian Space Agency (ASI).

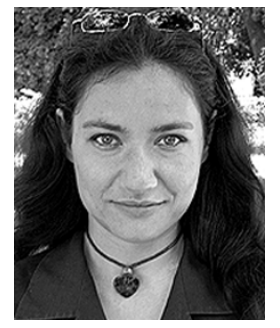

Raffaella Guida (S'04) was born in Naples, Italy, on October 24, 1975. She received the laurea degree (cum laude) in telecommunications engineering from the University of Naples "Federico II," Naples, Italy, in 2003. She is currently pursuing the Ph.D. degree in electronic and telecommunication engineering.

In 2003, she received a grant from University of Naples to be spent at the Department of Electronic and Telecommunication Engineering for research in the field of remote sensing. Her main research interests are in the field of microwave remote sensing, particularly in simulation and modeling of synthetic aperture radar signals relevant to natural surfaces as well as urban scenes.

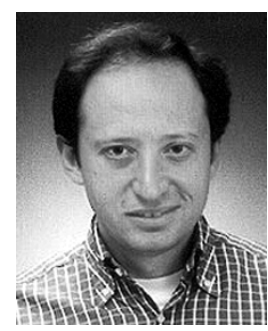

Antonio Iodice (S'97-M'00) was born in Naples, Italy, on July 4, 1968. He received the laurea degree (cum laude) in electronic engineering and the Ph.D. degree in electronic engineering and computer science, both from the University of Naples, in 1993 and 1999, respectively.

In 1995, he received a grant from CNR (Italian National Council of Research) to be spent at IRECE (Instituto di Ricerca per 1'Elettromagnetismo e i Componenti Elettronici), Naples, for research in the field of remote sensing. He was with the Department of Electronic and Telecommunication Engineering, University of Naples, from 1996 to 1999, and with Telespazio S.p.A., Rome, Italy, from 1999 to 2000. He is currently a Research Scientist at the Department of Electronic and Telecommunication Engineering, University of Naples, where he also teaches electromagnetics and guided propagation. His main research interests are in the field of microwave remote sensing: modeling of electromagnetic scattering from natural surfaces, simulation and modeling of SAR signals, and SAR interferometry. 


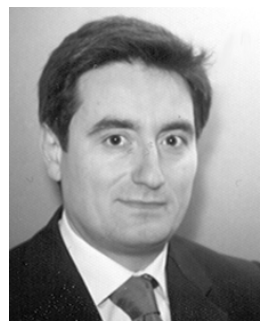

Daniele Riccio (M'91-SM'99) was born in Naples, Italy, on April 13, 1962. He received the laurea degree (cum laude) in electronic engineering from the University of Naples "Federico II," Naples, Italy, in 1989.

Currently, he is Professor of remote sensing, electromagnetic diagnostics, and guided propagation at the University of Naples "Federico II." He was a Research Scientist at the Istituto di Ricerca sull'Elettromagnetismo e i Componenti Elettronici (IRECE), Italian National Council of Research (CNR), and at the Department of Electronic and Telecommunication Engineering, University of Naples "Federico II." In 1994 and 1995, he was also a Guest Scientist at the DLR High-Frequency Institute, Munich, Germany. His research activity is witnessed by more than 110 papers published in the fields of microwave remote sensing, simulation and modeling of synthetic aperture radar signals relevant to terrestrial oceanic and urban scenes, as well as in the application of fractal geometry to electromagnetic scattering and remote sensing.

Prof. Riccio has won several fellowships from private and public companies (SIP, Selenia, CNR, CORISTA, and CRATI) for research in the remote sensing field.

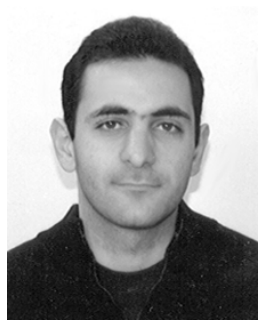

Giuseppe Ruello (S'00-M'04) was born in Naples, Italy, on February 12, 1975. He received the laurea degree (with honors) in telecommunication engineering and the Ph.D. degree in electronic and telecommunication engineering, in 1999 and 2003, respectively, both from the University of Naples "Federico II," Naples, Italy.

In 2000, he received a grant from the University of Naples to be spent at the Department of Electronic and Telecommunication Engineering for research in the field of remote sensing, and at the same time, he also won a grant from University of Rome "La Sapienza," Rome, Italy. In 2002, he was a Visiting Scientist at the Department of Signal Theory and Communications of the Universitat Politecnica de Catalunya, Barcelona, Spain. His main research interests are in the field of SAR remote sensing, modeling of electromagnetic scattering from natural surfaces, and SAR simulation. 